\title{
Bronchial Thermoplasty by Application of Ultrasound Energy
}

Lidia Sobkow ${ }^{1}$, Sajive Aleyas ${ }^{2}$, Matthew Pollman ${ }^{1}$, Dong lk Shin ${ }^{1}$, Joerg Schulze-Clewing ${ }^{1}$, Tom Zimmerman ${ }^{3}$, Valery Matthys ${ }^{4}$, Jean Rooney ${ }^{3}$, Li Liu ${ }^{4}$ and Reinhard J. Warnking ${ }^{1 *}$

${ }^{1}$ Guided Interventions Inc., 25 Health Sciences, Dr., Stony Brook, NY, USA

${ }^{2}$ Lung Cancer Evaluation Center, Department of Medicine, Stony Brook Medicine, USA

${ }^{3}$ Division of Laboratory Animal Resources Stony Brook University, USA

${ }^{4}$ Center for Biotechnology, an Empire State Development Division of Science Technology and Innovation (NYSTAR) Center for Advanced Technology, Bioengineering Building, 2nd Floor, Stony Brook University, Stony Brook, NY, USA

\begin{abstract}
Background: Asthma is a chronic inflammatory disease of respiratory airways, typically marked by spasms in the bronchi of the lungs, causing difficulty in breathing. Although the disease is very well documented, therapies are limited and patients mostly undergo symptom management. Bronchial thermoplasty is a radiofrequency-based treatment for severe asthma approved by Food and Drug Administration (FDA) in 2010 (Alair system, Boston Scientific Inc). Here we propose the use of ultrasound (US) energy to perform bronchial thermoplasty. Our hypothesis is that ultrasound affects bronchial smooth muscle at least as effective as radio-frequency (RF) but causes less collateral damage, and holds the potential to reduce the procedure time. In this publication we present a direct comparison of acute thermoplasty effects of RF energy versus ultrasound energy.
\end{abstract}

Objective: To evaluate a bronchoscopic procedure based on circumferential ultrasound energy and its effect on bronchial wall structures in comparison to RF thermoplasty for asthma treatment.

Methods: This is an observational study aiming for a direct comparison of an ultrasound system with existing asthma therapy based on radio-frequency. Mongrel dogs were used for optimization of ultrasound energy (300 J, $200 \mathrm{~J}, 100 \mathrm{~J}$ ) with a 4.5 and $5.5 \mathrm{~mm}$ balloon catheter. $100 \mathrm{~J}$ was ultimately directly compared with RF effects on bronchial wall structures. Safety and efficacy of the ultrasound system was already previously established during renal denervation clinica stdies.

Results: 12 mongrel dogs were used. Bronchoscopy procedures were feasible in all cases, and no adverse effects were seen in any dogs. Lungs were collected for histology and H\&E staining. With the optimized $100 \mathrm{~J}$ ultrasound energy we observed mild bronchial tissue injury, with minimal hemorrhage and preservation of respiratory epithelium. The RF catheter caused distinct focal injury points resulting from direct contact of the RF electrodes with bronchial wall tissue. Acute histological examination shows hemorrhagic injury along the RF energy path with significant necrosis to all bronchial wall elements including respiratory epithelium.

Conclusion: Ultrasound energy is safe, feasible, and at least as effective in affecting bronchoconstriction as RF energy offering a viable alternative to radiofrequency-based lung disease treatments.

Keywords: Bronchial thermoplasty; Ultrasound; Radiofrequency; Asthma; Comparative study; Bronchial wall; Feasibility

\section{Introduction}

Asthma is a chronic inflammatory disease of respiratory airways typically marked, among other symptoms, by spasms in the bronchi of the lungs, causing difficulty in breathing. Both genetic and environmental conditions are currently thought to cause asthma [1]. Although asthma is very well documented, precise diagnosis is still challenging and is usually based on multiple symptoms, patient history, response to therapy, and responsiveness to treatment [2-5].

Most patients undergo standard principles of treatment and symptom management: corticosteroids, long acting beta agonists, and elimination of asthma triggers $[3,4]$.

Almost 25 million Americans have asthma and approximately 10\% of them have been categorized as severe. American spending related to asthma reaches $\$ 18$ billion, the majority of which is due to emergency hospital visits and multiple medications [4]. Globally, 235 million people suffer from asthma, and it is considered the most common chronic disease among children [5].

Bronchial Thermoplasty (BT) is a treatment for severe asthma approved by FDA in 2010. It involves delivering controlled 10 second, $65 \mathrm{C}$ heat pulses to the bronchial wall and damaging smooth muscle sections within the bronchial wall. The only device currently available on the market is the Alair System (Boston Scientific), which relies on therapeutic effects of radio-frequency (RF) energy. The process includes three separate bronchoscopic procedures, performed three weeks apart(l). Upon treatment with BT, the numbers and severity of exacerbations are reduced. This procedure seems to have opened new avenues for treatment of severe asthma cases, and only very recently it has gained interest of insurers'. Boston Scientific published a 5 year follow up report [6] on patients treated with BT, observed during clinical studies where patients continued to take their standard maintenance medication. The report claims significant reduction in asthma attacks, ER visits, and hospitalizations for respiratory symptoms.

Here, we propose the use of ultrasound (US) energy to perform bronchial thermoplasty. The ultrasound system utilized was previously designed for renal denervation [7]. The proof of concept was obtained and basic safety and design parameters of this system were established previously through in vitro studies on 148 tissues specimens and in

*Corresponding author: Reinhard J. Warnking, Guided Interventions Inc., 25 Health Sciences Dr. Stony Brook, NY 11790, USA, Tel: 001-631-8391774; E-mail: rwarnking@guidedinterventions.com

Received July 21, 2014; Accepted October 15, 2015; Published October 19, 2015

Citation: Sobkow L, Aleyas S, Pollman M, Shin DI, Schulze-Clewing J, et al. (2015) Bronchial Thermoplasty by Application of Ultrasound Energy. J Pulm Respir Med 5: 293. doi:10.4172/2161-105X.1000293

Copyright: @ 2015 Sobkow L, et al. This is an open-access article distributed under the terms of the Creative Commons Attribution License, which permits unrestricted use, distribution, and reproduction in any medium, provided the original author and source are credited. 
vivo studies on 33 animals and showed clear potential of ultrasound to ablate distant nerve tissue with minimal or no injury to arterial tissue in porcine renal arteries [7].

Literature search (PubMed, Google Scholar, Web of Science, Google Patents) has shown that application of ultrasound for asthma or any other lung related condition has never been documented so far. In particular, no experiments have been documented with ultrasound to perform bronchial thermoplasty.

We hypothesized that ultrasound energy affects bronchial contractility and causes less damage to bronchial walls than RF. Moreover, due to application of a volumetric ultrasound energy field it might also be more effective, and can potentially be performed within one patient visit offering a significant reduction of overall burden of the bronchial thermoplasty procedure (including cost).

Since the acute procedural endpoint is the functional bronchial constriction in response to irritants this experiment cannot be performed ex vivo. A canine model has been used in previous experiments with RF energy [8]. Utilizing the same canine model allowed us to minimize the overall number of animals, since our observations could be directly compared with the previously conducted RF experiments.

\section{Materials and Methods}

\section{Animal care}

All personnel was cleared by the Department of Preventative Medicine and registered and approved by the Occupational Health Division of Stony Brook University. All experiments were performed with assistance of the Division of Laboratory Animal Resources (DLAR) personnel under the supervision of Dr. T. Zimmerman. All experiments were approved by the Stony Brook University's Institutional Animal Care and Use Committee (IACUC \# 2013-2079-NF-USDA-DA).

Anesthesia: For all treatments and follow up bronchoscopies, animals were anesthetized with propofol $(6-8 \mathrm{mg} / \mathrm{kg}, 20 \mathrm{ml}, \mathrm{IV})$ and the effect was maintained by isofluorane (1-5\%) administered during continuous, mechanical ventilation. During anesthesia heart rate, respiratory rate, response to noxious stimulus, movement were monitored every $15 \mathrm{~min}$.
Animals were subjected to preanesthetic fasting 18 hours prior to the procedure.

Animals: Twelve, 5 months-old female, dogs were purchased from Marshall BioResources by DLAR at Stony Brook University. Animals were quarantined at the DLAR for a week prior to the procedure and taken care of according to standard DLAR protocol.

Euthanasia as an endpoint: In order to collect lung samples and record effect of the ultrasound on bronchial wall and tissue surrounding bronchial wall (perform appropriate histology and comparison studies) we had to sacrifice animals via administration of pentobarbital overdose solution at $100 \mathrm{mg} / \mathrm{kg}$ via intravenous injection.

\section{Bronchoscopy procedure, baseline measurements, equipment}

The bronchoscopy procedure was done according to the protocol described in Danek et al. [8]. Briefly, the bronchoscope was introduced to a lung via tracheal tube and advanced to the lower (left and right) lobes. Ultrasound treatment was delivered bronchoscopically to the airways 24 hours post baseline metacholine $(\mathrm{MCh})$ challenge. Bronchoscopic observation, measurement of resting airway diameter, and airway responsiveness to local MCh challenge was performed before treatment (establishing baseline) and after 1 day for all dogs. $\mathrm{MCh}$ challenge was also performed at later time points before dogs were sacrificed (Table 1).

Bronchoscopy equipment used for the procedure: Bronchoscope Olympus BF-1T30, length $50 \mathrm{~cm}$, working channel diameter $2.8 \mathrm{~mm}$; Camera (pig tail) OVC100 Evis Olympus; Light source Olympus SLV160; Video processor Olympus SV160; Image recording with microphone Toshiba DVD recorder 1080P.

\section{Measurement of the resting diameter and airway responsiveness to local $\mathrm{MCh}$ challenge}

Resting airway diameter and airway responsiveness to the MCh challenge was measured at discrete sites within the bronchial tree, via visual navigation through the bronchoscope (Figure $1 \mathrm{~A}$ and $1 \mathrm{~B}$ ). Sites were mapped and video recorded. Baseline measurements and post treatment follow up sessions were recorded as well. Resting airway

\begin{tabular}{|c|c|c|c|c|c|c|c|c|c|c|c|c|c|c|c|c|}
\hline & \multicolumn{4}{|c|}{1 - experimental outline : } & \multicolumn{4}{|c|}{2 - experimental outline : } & \multicolumn{8}{|c|}{3 - experimental outline : } \\
\hline & \multicolumn{4}{|c|}{$\begin{array}{l}\text { Day 0: US+MCh dog 1-4 } \\
\text { Day 1: follow up; MCh challenge dogs } \\
\text { 1-4; euthanasia +tissue collection on } \\
\text { dog 1, } 2 \\
\text { Day 7: follow up; MCh challenge dogs } \\
3,4 ; \text { euthanasia +tissue collection on } \\
\text { dog } 3,4\end{array}$} & \multicolumn{4}{|c|}{$\begin{array}{l}\text { Day -1: baseline measurement } \\
\text { Day 0: US+MCh dog } 5,6 \\
\text { Day 1: US+MCh dog } 7,8 \\
\text { Day 7: day } 7 \text { follow up; } \mathrm{MCH} \text { challenge; } \\
\text { euthanasia +tissue collection on dog } 5,6 \\
\text { Day 21: day } 21 \text { follow up; } \mathrm{MCH} \\
\text { challenge; euthanasia +tissue collection } \\
\text { on dog } 7,8\end{array}$} & \multicolumn{8}{|c|}{$\begin{array}{l}\text { Day 0: US+RF+MCh dog9-12 } \\
\text { Day 1: follow up; MCh dog 9-12; euthanasia +tissue collection on dog } \\
9,10 \\
\text { Day 28: follow up; MCH challenge; euthanasia +tissue collection on dog } \\
\text { 11, } 12\end{array}$} \\
\hline & $\operatorname{dog} 1$ & $\operatorname{dog} 2$ & $\operatorname{dog} 3$ & $\operatorname{dog} 4$ & $\operatorname{dog} 5$ & $\operatorname{dog} 6$ & $\operatorname{dog} 7$ & $\operatorname{dog} 8$ & \multicolumn{2}{|l|}{$\operatorname{dog} 9$} & \multicolumn{2}{|l|}{$\operatorname{dog} 10$} & $\operatorname{dog} 11$ & & \multicolumn{2}{|l|}{$\operatorname{dog} 12$} \\
\hline & \multicolumn{4}{|c|}{$\begin{array}{l}\text { Suggested as many ablations per lobe } \\
\text { as possible. }\end{array}$} & \multicolumn{2}{|c|}{$\begin{array}{l}\text { suggested } 6 \\
\text { lesions per lobe, } \\
\text { we targeted left } \\
\text { and right lower } \\
\text { lobes }\end{array}$} & \multicolumn{2}{|c|}{$\begin{array}{l}\text { 2- } 6 \text { lesions per lobe } \\
\text { to test global effect } \\
\text { of denervation with a } \\
\text { compliant balloon }\end{array}$} & \multicolumn{8}{|c|}{$\begin{array}{l}\text { To compare RF system to optimized ultrasound system. } \\
\text { * - temperature controlled energy application. For details see RF } \\
\text { manufacturer's operations manual. }\end{array}$} \\
\hline $\begin{array}{l}\text { balloon } \\
\text { size }\end{array}$ & $4.5 \mathrm{~mm}$ & $4.5 \mathrm{~mm}$ & $4.5 \mathrm{~mm}$ & $5.5 \mathrm{~mm}$ & $4.5 \mathrm{~mm}$ & $4.5 \mathrm{~mm}$ & $10 \mathrm{~mm}$ & $10 \mathrm{~mm}$ & $5.5 \mathrm{~mm}$ & RF & $5.5 \mathrm{~mm}$ & RF & $5.5 \mathrm{~mm}$ & RF & $5.5 \mathrm{~mm}$ & RF \\
\hline $\begin{array}{l}\text { treatment } \\
\text { setting }\end{array}$ & $\begin{array}{l}10 W \\
30 s \text { RL; } \\
10 W \\
20 \text { s LL }\end{array}$ & $\begin{array}{l}10 \mathrm{~W} \\
20 \mathrm{~s} \text { RL; } \\
10 \mathrm{~W} \\
20 \mathrm{~s} \mathrm{LL}\end{array}$ & $\begin{array}{l}10 \mathrm{~W} \\
10 \mathrm{~s} \text { RL; } \\
10 \mathrm{~W} \\
10 \mathrm{~s} \text { LL }\end{array}$ & $\begin{array}{l}\text { 10W, 10s } \\
\mathrm{RL} ; 10 \mathrm{~W} \\
10 \mathrm{~s} \text { LL }\end{array}$ & $\begin{array}{l}10 W \\
15 s \text { RL; } \\
10 W \\
20 \text { s LL }\end{array}$ & $\begin{array}{l}10 \mathrm{~W} \\
15 \mathrm{~s} \text { RL; } \\
10 \mathrm{~W} \\
20 \mathrm{~s} \text { LL }\end{array}$ & $\begin{array}{l}10 \mathrm{~W} \\
15 \mathrm{~s} \text { RL; } \\
10 \mathrm{~W} \\
20 \mathrm{~s} \mathrm{LL}\end{array}$ & $\begin{array}{l}10 \mathrm{~W}, 15 \mathrm{~s} \\
\mathrm{RL} ; 10 \mathrm{~W} \\
20 \mathrm{~s} \text { LL }\end{array}$ & $\begin{array}{l}\text { US;LL } \\
-10 W \\
10 s\end{array}$ & $\begin{array}{l}\mathrm{RF} ; \mathrm{RL} \\
-*\end{array}$ & $\begin{array}{l}\text { US;LL } \\
-10 W, \\
10 s\end{array}$ & $\begin{array}{l}\mathrm{RF} ; \\
\mathrm{RL} \text { - * }\end{array}$ & $\begin{array}{l}\text { US;LL } \\
-10 W \\
10 s\end{array}$ & $\begin{array}{l}\mathrm{RF} ; \mathrm{RL} \\
-*\end{array}$ & $\begin{array}{l}\text { US;LL } \\
-10 W \\
10 s\end{array}$ & $\begin{array}{l}\mathrm{RF} ; \mathrm{RL} \\
-*\end{array}$ \\
\hline \# lesions & \multicolumn{2}{|c|}{ total 27 ablations } & $\begin{array}{l}\text { 16RL; } \\
\text { 8LL }\end{array}$ & 1RL; 2LL & $\begin{array}{l}2 \mathrm{RL} ; \\
\text { 9LL }\end{array}$ & $5 \mathrm{RL}$ & $\begin{array}{l}2 \mathrm{RL}, 4 \\
\mathrm{LL}\end{array}$ & $2 \mathrm{RL} ; 6 \mathrm{LL}$ & 6 & 6 & 6 & 6 & 7 & 8 & 8 & 6 \\
\hline
\end{tabular}

$\mathrm{RF}$ - radio-frequency; US - ultrasound, $\mathrm{RL}$ - right lung; $\mathrm{LL}$ - left lung, $\mathrm{MCh}$ - metacholine, $\mathrm{W}$ - Watt, s - second.

Table 1: Detailed experimental design of ultrasound vs. radio-frequency bronchial thermoplasty studies. 

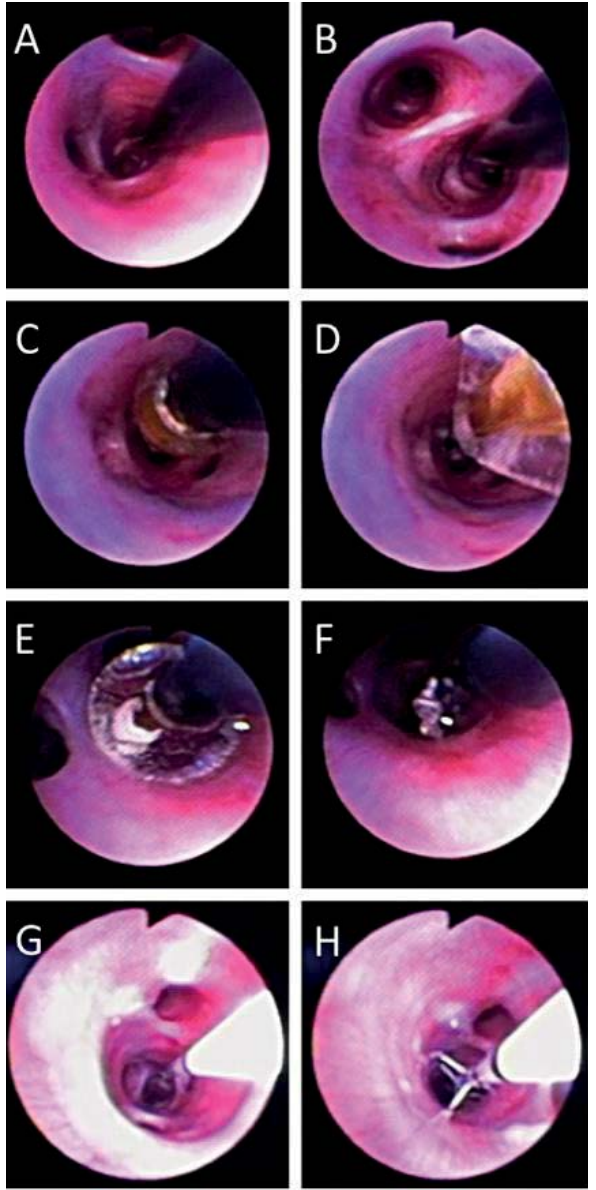

Figure 1: Measurement of the resting diameter and airway responsiveness to local MCh challenge and visual representation of catheters used in the study. Bronchial diameter measurements at resting state (A, B). Inflated (C) and deflated (D) $4.5 \mathrm{~mm}$ balloon. Inflated (E) and deflated (F) compliant 10 $\mathrm{mm}$ balloon. G: Alair catheter shaft with navigation markings; $\mathrm{H}$ : expanded radio-frequency electrodes at the tip of Alair catheter touching bronchial walls.

diameter was measured using the catheter shaft as a visual reference (Figure 1A and 1B). The US catheter shaft is $2.0 \mathrm{~mm}$ in diameter (Figure $1 \mathrm{~A}$ and $1 \mathrm{~B}$ ), with the transducer at the distal tip surrounded by a balloon with an expanded diameter of 4.5 or $5.5 \mathrm{~mm}$ (Figure $1 \mathrm{C}$ and 1D) or compliant up to $10 \mathrm{~mm}$ diameter balloon (Figure $1 \mathrm{E}$ and $1 \mathrm{~F}$ ). The diameter of the airway was visually compared to the diameter of the catheter shaft. Sites selected for treatments were 3-6 $\mathrm{mm}$ in size for the 4.5-5.5 $\mathrm{mm}$ balloon sizes and larger bronchi for the $10 \mathrm{~mm}$ compliant balloon.

Baseline MCh response: After resting airway diameter measurement, MCh (Acetyl- $\beta$-methylcholine chloride $\geq 98 \%$ (TLC), powder, Sigma) was sprayed onto the airway site $(0.025 \mathrm{mg} / \mathrm{ml}$ in sterile normal saline, approx. $100 \mu \mathrm{l})$. See Figure 2A-2D.

\section{Histology}

McClain Laboratories, Smithtown NY performed histological slide preparation and basic examinations of the postmortem lung specimens. Lungs 1 and 2 were fixed in 10\% formalin solution over the weekend. Lungs 3 and 4 were fixed in 20\% formalin for $48 \mathrm{~h}$. Lungs 5-12 were fixed in $10 \%$ formalin solution overnight. Lower lobes were separated from the lung tree and marked for orientation. Each specimen was sliced to a number of $5 \mathrm{~mm}$ slices and photographed as a gross image (example - Figure 3A, 3E, 4A and 4E). Transverse sections of each site were fixed and stained with hematoxylin and eosin. Examination of the tissue allowed us to pinpoint lesion sites and reconstruct the affected bronchi. Images were taken with a standard Zeiss light microscope.

\section{Ultrasound and Radio-frequency equipment}

Ultrasound (US): The catheter design used for this procedure was previously tested for safety and efficacy in renal denervation trials on pigs. Three catheter models with different balloon designs were used ( $4.5 \mathrm{~mm}, 5.5 \mathrm{~mm}$ and a compliant balloon expanding up to $10 \mathrm{~mm}$ - see Figure $1 \mathrm{C}-1 \mathrm{~F}$ ) allowing for different energy dispersion profiles. Catheters are designed to allow for water flow through a balloon in order to provide both cooling to the transducer as well as a medium for the energy transmission from the transducer to the surrounding tissue.

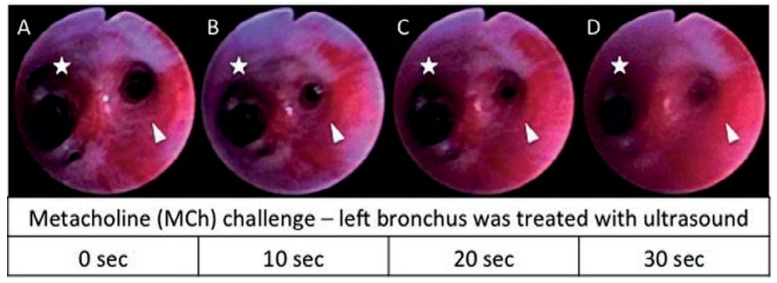

Figure 2: Metacholine (MCh) challenge. A-D show progression of MCh induced bronchoconstriction of US treated (star) and untreated (arrowhead) bronchus. Response was recorded within 30 second window and was consistent throughout the whole study for US and RF treated bronchi.

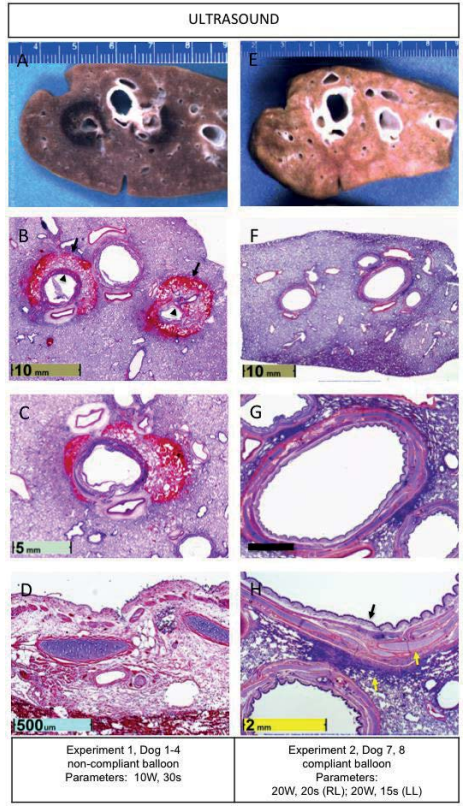

Figure 3: General observations after gross examination and histological analysis of acute injuries from animals treated with different US doses. A-D represents initial US dose effect on lung tissue used in Experiment 1, dog 1-4. 300 Joules US dose $(10 \mathrm{~W}, 30 \mathrm{sec})$ resulted in clear, up to $5 \mathrm{~mm}$ deep hemorrhagic rings around bronchi $(A)$ and necrosis of all bronchial wall elements in the energy path (B-D). 2B: arrowheads are indicating damaged bronchial wall; arrowheads are indicating the extent of damage caused by the signal. $\mathrm{E}-\mathrm{H}$ shows selective nerve ablation in larger proximal bronchi treated with a compliant balloon of up to $10 \mathrm{~mm}$ diameter after 21 day follow up (Experiment 2, dog 6-7). Ultrasound dose was optimized for the balloon size and US dispersion $(\mathrm{E}-\mathrm{H}) .2 \mathrm{H}$ : black arrow indicates healthy bronchial epithelium, whereas yellow arrows are pointing at signs of inflammation (left yellow arrow) and scarring around necrotic cartilage (right yellow arrow). W: Watt, s: Second; RF: RadioFrequency. 

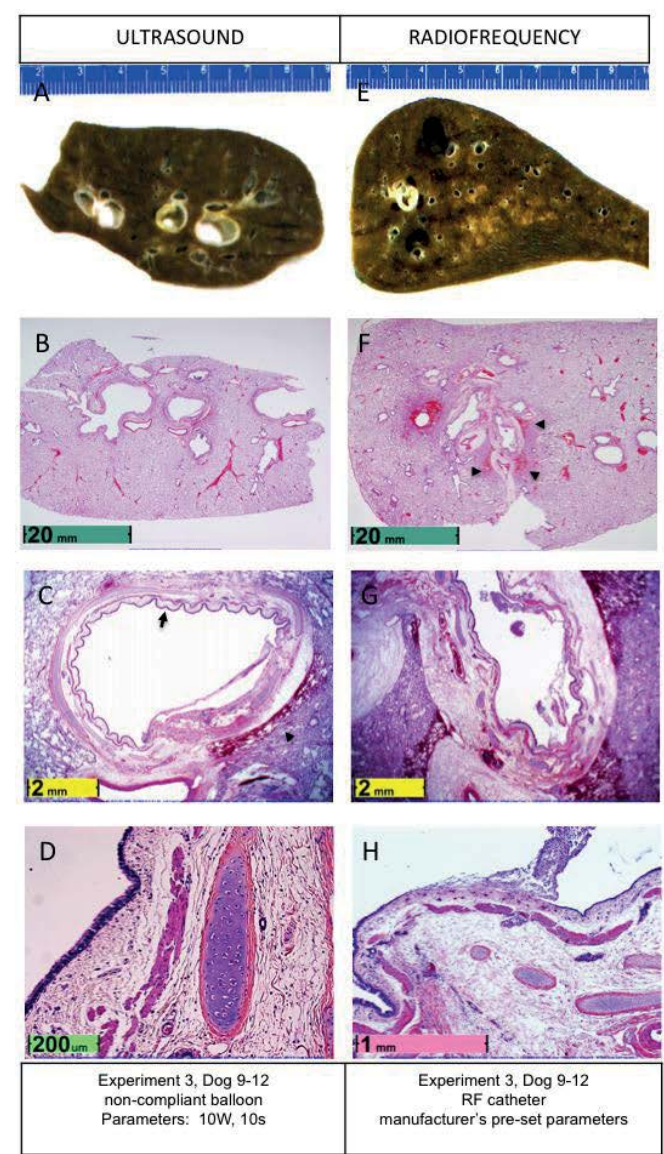

Figure 4: General observations after gross examination and histologica analysis of acute injuries from animals treated with optimized ultrasound dose and RF (manufacturer's pre set parameters). Experiment 3, dog 9-12. (A-D) Selection of $100 \mathrm{~J}$ energy dose of $10 \mathrm{~W}, 10 \mathrm{sec}$ allowed for a more differentiated injury model targeted mainly at nerve and muscle injury. We observe mild bronchial tissues injury, with minimal hemorrhage (arrowhead in $3 \mathrm{C}$ ) and preservation of respiratory epithelium (arrow in $3 \mathrm{C}$ ). (E-H) Acute effect of the Alair system delivering radio-frequency energy. Due to catheter design and pre set energy levels, there are distinct focal injury points resulting from direct contact of catheter electrode with bronchial wall tissue (arrowheads in $3 \mathrm{~F}$ ). Acute injury shows hemorrhagic injury along the energy path with significant necrosis to all bronchial wall elements including respiratory epithelium. RL: Right Lung; LL: Left Lung; W: Watt, s: Second

(Figure 5 - energy dispersion of ultrasound vs RF).

The Therapeutic Ultrasound System is comprised of the following components: Ultrasound Catheter, Ultrasound Console, Tubing Set, Connection Cable.

Radio-frequency (RF): Alair ${ }^{\mathrm{m}}$ Bronchial Thermoplasty System, Boston Scientific. (Figure $1 \mathrm{G}$ and $1 \mathrm{H}$ show the Alair catheter inside a bronchus).

\section{Results}

The goal of this comparative and observational study is to demonstrate feasibility of ultrasound to affect bronchoconstriction. The experiments described here were designed for a direct comparison of ultrasound (US) energy to existing radio-frequency (RF) technology used for bronchial thermoplasty, Alair (Boston Scientific), approved by FDA in 2010. The experiments compare preclinical studies for the RF

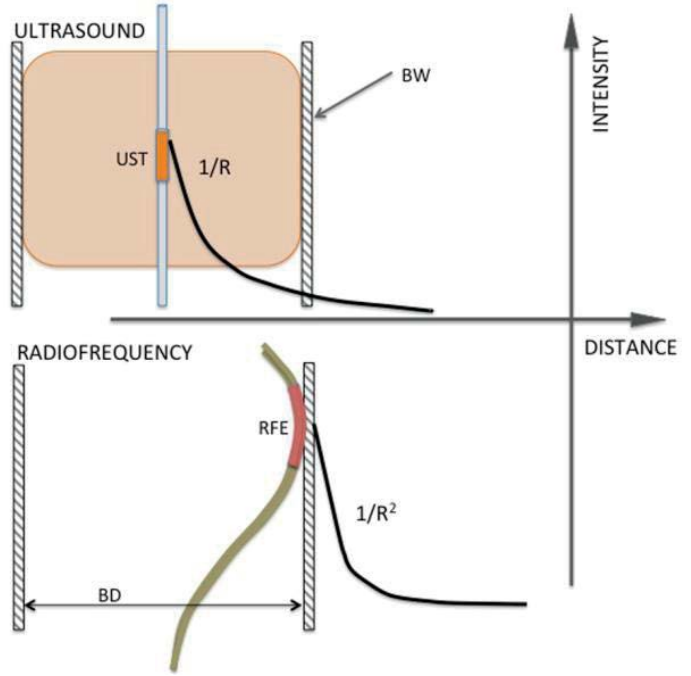

Figure 5: Graphic representation of energy dispersion and profiles for ultrasound and radio-frequency catheters. Abbreviations: UST: Ultrasound Transducer; RFE: Radiofrequency Electrode; BW: Bronchial Wall; BD: Bronchial Diameter.

system [8] with ultrasound studies in order to minimize the number of animals required.

We designed our study in 3 successive experiments (detailed parameters in Table1).

Experiment 1 was intended to test parameters previously established for the ultrasound system in renal denervation [7]. We used 4 animals to establish dosimetry and confirm the optimal US dose required affecting lung tissue and study adverse effects. No adverse effects like bronchospasm were observed.

Experiment 2 was designed to test functionality of a compliant balloon surrounding the transducer and the possibility of global (whole lung) denervation at the level of the main carina ( 2 animals). We used the additional two animals to confirm the optimal energy dose established in Exp. 1 for the given balloon diameters used in the study.

Experiment 3 was designed for direct comparison of the ultrasound system to the Alair system in bronchial thermoplasty procedures (4 animals).

In experiment 1 we started by evaluating the energy dose optimized for renal denervation [7]. We used 4 mongrel dogs and tested three distinct energy levels to determine any adverse effects, like bronchospasm and to measure the most effective and least damaging energy value. Step one used two animals (Dog 1 and 2). We tested two energy settings (10 Watt acoustic/30 sec and 10 Watt acoustic/20 sec) to observe the acute effect of ultrasound energy (1 day post treatment). Step two used 2 animals to establish short-term effects of ultrasound energy (1 week post treatment - Dog 3 and 4). Based on observations from the first two animals (see gross and histology in Figure 3A-3D) we decided to lower the energy output to 10 Watt acoustic for $10 \mathrm{sec}$. Each animal at their respective time point was euthanized; lungs were collected and sent for histopathological analysis (E\&H staining).

To evaluate the functional effect of US on bronchial constriction we applied metacholine (MCh) to US-treated and untreated (control) bronchi $15 \mathrm{~min}$ after the US treatment. We observed minimal acute 
(immediate post experiment, or 1 day post treatment) response in sites treated with US, suggesting that the US treatment affected smooth muscle and its ability to contract (Figure 2A-2D). This response was consistent throughout the whole study.

Histological analysis indicates that acute and short term effects of the initial energy parameters (10 Watt acoustic/30 sec and 10 Watt acoustic/20 sec) cause too much immediate damage, affecting not only neural tissue but also muscle, cartilage and connective tissue as deep as $5 \mathrm{~mm}$ outside of the bronchial wall (Figure 2A-2D). In dogs 1-6 we consistently observed signs of inflammation and necrosis of cartilage, nerve twigs and muscle. Arteriole exhibit signs of healing thrombosis and atrophy of small nerve twigs. Extend of injury reaches 3-4 mm into the tissue. By day 21 we observe bronchial wall healing, expected at this time point. Energy of 100 Joules caused the desired lesion depth of only 1-2 $\mathrm{mm}$ around the bronchial wall. We tested those parameters on two additional animals $(\operatorname{dog} 5,6)$ and confirmed that $100 \mathrm{~J}(10 \mathrm{~W}, 10 \mathrm{sec})$ dose is an optimal dose given radius ( $\mathrm{r}$ ) of this catheter design $(4.5 \mathrm{~mm}$ balloon diameter) for bronchial thermoplasty (Figure 4A-4D).

We also tested the compliant balloon with the parameters of $10 \mathrm{~W}$, $15 \mathrm{sec}$ in the Right Lung of $\operatorname{dog} 7$ and 8; $10 \mathrm{~W}, 20 \mathrm{sec}$ Left Lung of dog 7 and 8 . This was designed to evaluate the denervation level obtained by the ultrasound energy. Animals were sacrificed after 21 days (Figure $3 \mathrm{E}-3 \mathrm{H})$ to observe possible healing processes and to observe impact on nerves and whether we can maintain the denervation effect after 3 weeks. Histological evaluation of affected lung tissue revealed signs of inflammation with scarring around necrotic cartilage. Small nerves present in the zone of injury exhibit signs of healing with reduced numbers of axons (Figure 3E-3H).

We treated proximal bronchi with the expectation that one proximal lesion will affect the functional response of bronchi distal to the treatment site (bronchoconstriction in response to $\mathrm{MCh}$ ). However, with the Metacholine challenge we did not observe this effect and we cannot at this point conclude whether we were able to induce a global denervation effect by targeting nerve bundle proximally (e.g. as in vagotomy described by Dimitrov-Szokodi et al. [9]). The MCh model turned out to be not appropriate to test for proximal denervation. Metacholine is an agonist of muscarinic receptors, and its presence in lungs will mimic activation of the parasympathetic nervous system [10]. MCh (as acetylocholine) will bind to muscarinic receptors on smooth muscle of a bronchus, causing activation of a PLC pathway (phospholipase $\mathrm{C}$ pathway regulating calcium levels in the cell) and ultimately causing muscle constriction. Hence, when nerves proximal to the MCh application were disrupted (hopefully disabling all downstream nerve signal transmission) it would not affect local response of smooth muscles of bronchus to metacholine challenge. Therefore, separate studies with a more appropriate denervation measurement would have to be performed.

Finally, we compared US directly with the RF. The goal was to compare optimized ultrasound parameters to commercially available and in clinical use, RF parameters. Four dogs were treated with 100 Joule ultrasound energy to the left lung and manufacturer's pre set RF dose to the right lung. Two dogs $[9,10]$ were sacrificed after one day post-treatment to observe acute effects of energy on bronchial wall and surrounding tissue. Two dogs were sacrificed after 28 days post US/RF treatment and evaluated histologically for signs of healing, regeneration and overall long-term differences between effects of US in comparison to RF. We observed mild bronchial tissue injury, with minimal hemorrhage and preservation of respiratory epithelium when US was used (Figure 4A-4D). The RF catheter delivering radio- frequency (Figure $4 \mathrm{E}-4 \mathrm{H}$ ) causes distinct focal injury points resulting from direct contact of the RF electrodes with bronchial wall tissue. Acute histological examination shows hemorrhagic injury along the energy path with significant necrosis to all bronchial wall elements including respiratory epithelium.

\section{Discussion}

No adverse events like broncho-spasm were observed in ultrasound or RF treated bronchi. Even in the initial ultrasound dose optimization experiments, with $300 \mathrm{~J}$ and $200 \mathrm{~J}$ respectively, no acute adverse effects were observed. Surprisingly, these initial energy levels caused very deep lesions which they did not in renal denervation [7]. Obviously, the ultrasound attenuation of lung tissue must be significantly lower than connective and fat tissue surrounding the renal arteries. A dose of $100 \mathrm{~J}$, as used in dogs 9-12, seems appropriate to optimize a selective ultrasound effect on mainly nerve and muscle tissue when utilizing a $5.5 \mathrm{~mm}$ balloon (Figure 4A-4D). An optimal ultrasound system design, of course, will utilize varying energy levels based on bronchial diameter. Based on the here described experiments an ultrasound system optimized for bronchioplasty would employ a highly compliant balloon with variable diameter and a diagnostic mode to detect the actual bronchial diameter. The diameter could be determined by sending out a short diagnostic pulse through the cylindrical ultrasound transducer. In a perfectly round bronchus a short echo will be received after a certain time interval, $\Delta \mathrm{t}$, which is proportional to the bronchial diameter. In an oval structure the echo signal will have a certain length l, determined by $\mathrm{d}_{\max }$ minus $\mathrm{d}_{\min }$. The energy level would be selected for example through a look up table based on $\left(\mathrm{d}_{\max }+\mathrm{d}_{\min }\right) / 2$. Also, some mechanical catheter design changes are required. In experiment 3 some hemorrhage could be observed which might have been caused by mechanical damage at the catheter tip. Since for bronchioplasty an over-the-wire (OTW) design is not required the current catheter tip can easily be eliminated and replaced by an atraumatic, blunt catheter tip.

Given the circumferential ultrasound field, and in this case a $6 \mathrm{~mm}$ length of the transducer a significantly greater volume can be treated with ultrasound with a single energy application compared to RF. This holds the potential to simplify the procedure and to possibly perform the treatment in one session rather than 3 sessions. Also, the minimal bronchial wall injury compared to RF lends itself to shorten the overall procedure time. Consistently, in the optimized dose experiments (4 dogs 9-12, see Figure 4A-4D) the bronchial epithelium is spared but muscle, nerve and cartilage are necrotic. This is not the case with RF (see Figure $4 \mathrm{E}-4 \mathrm{H}$ ) where epithelium is necrosed and tissue on the path of energy is affected including nerve, cartilage, epithelium, blood vessels, and muscle. This also becomes apparent in the gross examination, where US wounds are almost invisible (with optimized energy dose in experiment 3, (Figure 4A)) whereas RF wounds are very clearly depicted (acute), Figure 4E. In both cases (RF and US), also collagen structures are affected.

The most significant advantage of ultrasound, though, would be in targeting deeper lying structures like nerves, since the RF power intensity decays with $1 / \mathrm{r}^{2}$ while the ultrasound energy is following a $1 / \mathrm{r}$ curve, see Figure 5 . Also, with ultrasound, the peak intensity is located inside the cooling balloon and therewith kept away from the endothelium. An almost constant power distribution radially beyond the bronchial wall is the result (Figure 5 upper graph). This makes ultrasound energy more appropriate for deep lesion generation i.e. cancer treatment.

Alair is keeping the temperature at the source (electrode) at 65 degrees $\mathrm{C}$ through thermocouple control of the generator. Although, 
ultrasound is generating a more homogeneous power distribution, it might not be significantly advantageous in smooth muscle ablation due to the short distance between the target and the energy source. It certainly is of great advantage when deeper structures like renal nerves are targeted (5 to $10 \mathrm{~mm}$ from renal artery wall [7]). Targeting renal nerves with RF causes significant overheating of the renal intima.

In order to take full advantage of the above, described ultrasound characteristics deeper structures would need to be targeted. Bronchial nerves are located 2 to 3 times as deep as smooth muscle. If nerve ablation would inhibit bronchial contraction [11] there might be a potential for an easier much faster thermoplasty procedure with ultrasound.

Another application taking advantage of the ultrasound energy dispersion would be lung tumor ablation. 3D mapping systems i.e. Super Dimensions allow precise tumor localization in the lung. These systems combine 3D imaging with the localization of guide wires during bronchoscopy. Today these procedures are limited to taking targeted tissue samples before surgical tumor removal. As demonstrated in this study precise lesion volumes can be created bronchoscopically with ultrasound inside the lung. As described here, the advantage of ultrasound-generated lesions is the significant depth without undue near field (in this case endo-bronchial) damage. The 3D navigation system would be utilized to localize a suspicious tissue volume and to determine the optimal access pathway for the guide wire. A sheath or working channel with dilator would be advanced over the guide wire. Once the area of interest has been penetrated, the dilator would be withdrawn and the ultrasound catheter advanced into the lesion. Depending on lesion volume the ultrasound dose will be calculated and one or more lesions will be generated. The proposed treatment method could be combined with immediate biopsy or other diagnostic measurements.

\section{Acknowledgments}

This work was supported by the Center for Biotechnology, an Empire State
Development Division of Science, Technology and Innovation (NYSTAR) Center for Advanced Technology.

\section{Conflict of Interest Statement}

RJW, LS, MP, DIS, JSC are either employees or consultants for Guided Interventions.

\section{References}

1. Martinez FD (2007) Genes, environments, development and asthma: a reappraisal. Eur Respir J 29: 179-184.

2. Lemanske RF Jr, Busse WW (2010) Asthma: clinical expression and molecular mechanisms. J Allergy Clin Immunol 125: S95-102.

3. National Heart, Lung and Blood Institute (NHLBI) (2007) National Asthma Education and Prevention Program, Expert Panel Report 3: Guidelines for the Diagnosis and Management of Asthma.

4. Global Strategy for Asthma Management and Prevention, Global Initiative for Asthma (GINA).

5. WHO (2007) sthma.

6. Wechsler ME, Laviolette M, Rubin AS, Fiterman J, Lapa e Silva JR, et al. (2013) Bronchial thermoplasty: Long-term safety and effectiveness in patients with severe persistent asthma. J Allergy Clin Immunol 132: 1295-1302.

7. Neuzil P, Petru J, Vondrakova D, Kopriva K, Chovanec M, et al. (2012) TCT356 Circumferential Therapeutic Ultrasound for the Treatment of Resistan Hypertension: Preliminary Results of Human Feasibility Study (SOUND-ITV). J Am Coll Cardiol.

8. Danek CJ, Lombard CM, Dungworth DL, Cox PG, Miller JD, et al. (2004) Reduction in airway hyperresponsiveness to methacholine by the application of RF energy in dogs. J Appl Physiol (1985) 97: 1946-1953.

9. Balogh G, Dimitrov-Szokodi D, Husveti A (1957) Lung denervation in the therapy of intractable bronchial asthma. J Thorac Surg 33: 166-184.

10. Scott GD, Fryer AD (2012) Role of parasympathetic nerves and muscarinic receptors in allergy and asthma. Chem Immunol Allergy 98: 48-69.

11. Slebos DJ, Klooster K, Koegelenberg CF, Theron J, Styen D, et al. (2015) Targeted lung denervation for moderate to severe COPD: a pilot study. Thorax 70: 411-419. 